\title{
Disseminated Tuberculosis Presenting as Mesenteric and Cerebral Abscess in HIV Infection: Case Report
}

\author{
Vinay Pandit ${ }^{1}$, Rohith Valsalan ${ }^{2}$, Shubha Seshadri ${ }^{3}$ and Shibu Bahuleyan ${ }^{2}$ \\ ${ }^{1}$ Associate Professor, Department of Medicine, Kasturba Medical College; ${ }^{2}$ Assistant Professor, Department of Medicine, Kasturba Medical \\ College; ${ }^{3}$ Professor, Department of Medicine, Kasturba Medical College; Manipal, India
}

\begin{abstract}
Disseminated tuberculosis in HIV infection involves multiple organs. Pulmonary and lymph node involvement are the commonest form of tuberculosis in HIV infection [1, 2]. Other forms of tuberculosis in the absence of lung and lymph node involvement are rare. Various forms of abdominal [3, 4] and neurological [5, 6] tubercular involvement in HIV infection have been reported. But tuberculosis presenting simultaneously with mesenteric and brain abscess has not been reported yet. We report a case of disseminated tuberculosis presenting as mesenteric and cerebral abscess in a HIV case without involving lung and lymph nodes. Bone marrow smears and fine needle aspiration cytology (FNAC) from mesenteric lesion were positive for acid fast bacilli (AFB) and the diagnosis of tuberculosis was confirmed by positive polymerase chain reaction (PCR). He responded well to treatment with anti tubercular drugs.
\end{abstract}

Key-Words: HIV, tuberculosis, abscess.

Tuberculosis is the most common opportunistic infection in HIV individuals in developing countries. The clinical presentation of tuberculosis varies depending on the severity of immunosuppression. In the early stages of HIV infection pulmonary involvement is most common; with progressive immunosuppression, extra pulmonary involvement presenting as lymphadenitis is most common [2]. Other organs involved in tuberculosis with advanced HIV disease include central nervous system, soft tissue, bone marrow, liver and other viscera. The disseminated tuberculosis, defined as having more than one focus or progressive hematogenous spread is reported in 38\% of cases [1]. Abdominal involvement is common in disseminated tuberculosis, especially lymphnode involvement. Abdominal sonographic abnormality is found in nearly $50 \%$ of cases of HIV infection [7]. Central nervous system tuberculosis occurs in 5\%-20\% of HIV-infected patients with tuberculosis and is primarily due to reactivation of latent infection or as a complication of disseminated disease. In HIV infected patients, with mild immunosuppression, histopathology will show typical granuloma with few or no AFB. But with severe degree of immunosuppression, there will be little cellular reaction with many AFB.

\section{Case Report}

Mr. HM, 42 year old male, agriculturist, was admitted with history of vomiting for six months, with a frequency of four to six times per day, non bilious and containing whatever he has consumed. His appetite was reduced. He had noticed right lower abdominal pain and fullness for one month. There was

Received on 5 April 2009; revised 13 July 2009.

Address for correspondence: Dr. Vinay Pandit. Associate Professor, Department of Medicine, Kasturba Medical College, Manipal - 576104, Karnataka, India. Phone: (91 0820) 2922513, 09844175598, Fax (91 820) 2571934. E- mail: vinayrpandit @gmail.com.

The Brazilian Journal of Infectious Diseases

2009;13(5):383-386. (C) 2009 by The Brazilian Journal of Infectious Diseases and Contexto Publishing. All rights reserved. an associated weight loss of ten kgs over six months and a low grade fever. There was no history of cough and expectoration, no past history of tuberculosis and he was not an alcoholic or a smoker. Examination showed body mass index of $18 \mathrm{~kg} / \mathrm{m}^{2}$ with normal vitals. He had glossitis and vitiligo without any lymphadenopathy. Abdominal examination revealed tender palpable mass of size $4 \times 4 \mathrm{~cm}$ in right illiac fossa, which was soft in consistency with ill defined borders. On Examination other systems were normal found to be normal. During the hospital stay he developed a left sided focal seizure with secondary generalization. Investigations revealed $\mathrm{Hb}-$ $8.5 \mathrm{gm} / \mathrm{dL}$, total WBC count $3.9 \times 10^{9}$ cell $/ \mathrm{L}$, platelet count $357 \times 10^{9} / \mathrm{L}$. Renal function tests and serum electrolytes were normal. Liver function test results were as follows - total protein- $7.9 \mathrm{gm} / \mathrm{dL}$, albumin -2.7gm/dL, globulin- $5.2 \mathrm{gm} / \mathrm{dL}$, AST - 57 U/L, ALT- 35U/L, Alkaline phosphate -22U/L. Hbs Ag was negative. ELISA and Western Blot for HIV were positive. Chest $\mathrm{X}$ - ray and CT scan of chest were normal. Upper GI endoscopy showed esophageal candidiasis with gastritis. Ultrasound scan of abdomen showed hypoechoic heterogeneous mass in right lumbar region with thickened bowel loops and thickened adjacent mesentry with interloop ascites. CT scan of abdomen showed multiseptated peripherally enhancing hypodense lesion in mesentry extending inferiorly to right illiac fossa and superiorly close to proximity of rectus muscle suggestive of mesenteric abscess. Thickening of distal jejunum, ileal loops and iliocecal junction with dilatation of proximal loops, and an associated omental thickening was noticed (Figures 1-2). CT scan of the brain was done and it showed a ring enhancing hypodense lesion with an enhancing mural nodule in right frontal lobe with surrounding perilesional edema and mass effect and effacement of adjacent sulci suggestive of tubercular abscess (Figures 3-4). CT guided FNAC from the abdominal lesion was done and the smear was consistent with abscess with good cellularity showing neutrophils, lymphocytes and histiocytes. PCR of the pus for tuberculosis was positive. Pus staining for AFB (acid fast bacillus) was positive. Bone marrow aspiration showed 
Figure 1. CT scan of abdomen showing hypodense lesion in the mesentry with thick surrounding capsule, multiple septations,thickening of adjacent bowel loops and omentum suggestive of tubercular abscess.

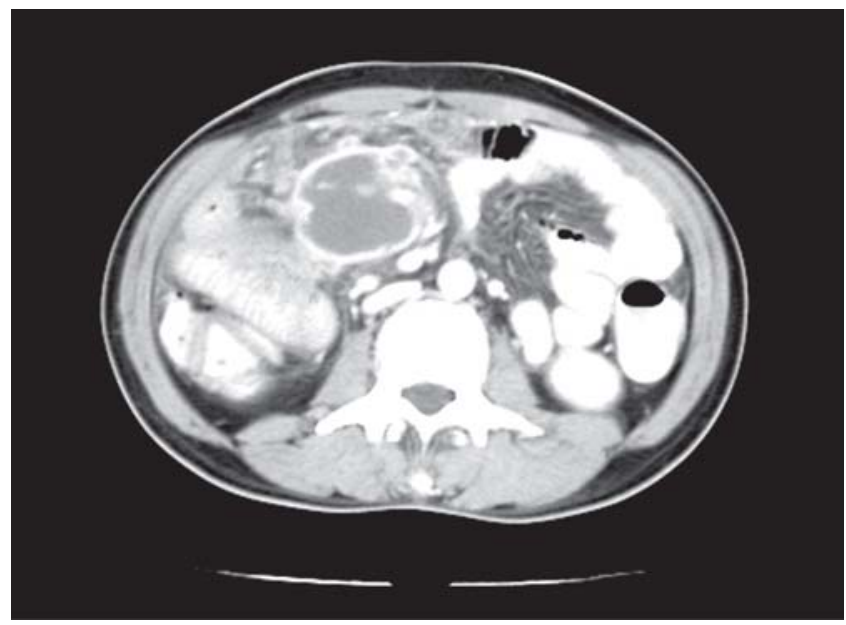

Figure 3. CT scan of brain showing ring enhancing, hypodense lesion in right frontal lobe and surrounding edema suggestive of tubercular abscess.

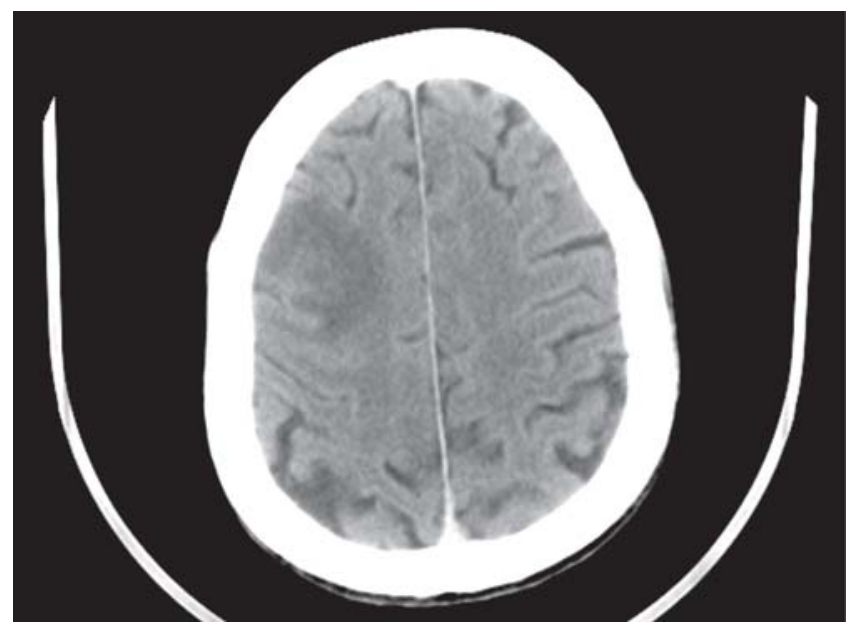

reactive forms with plasma cells $8 \%$, lymphocytes $10 \%$, eiosinophils $05 \%$ with occasional bands and dyspoetic forms. Marrow aspirate was also positive for AFB. His CD4 count was 124 cell $/ \mu l$. Toxoplasma IgM antibody and cryptococal antigen were negative. He was treated with fluconozole for ten days for candidiasis and antitubercular drugs for 12 months. On follow up, he showed good response to treatment and repeat investigations revealed resolution of abdominal lesion.

\section{Discussion}

The immune defect produced by HIV infection influences the natural history of tuberculosis. Over $15 \%$ of tuberculosis patients are likely to be HIV positive. In HIV infected individuals incidence of TB is nearly hundred folds compared to general population. Annual risk of developing clinical
Figure 2. CT scan of abdomen showing hypodense lesion in the mesentry with anterior extension up to rectus abdominal muscle and anterior abdominal wall.

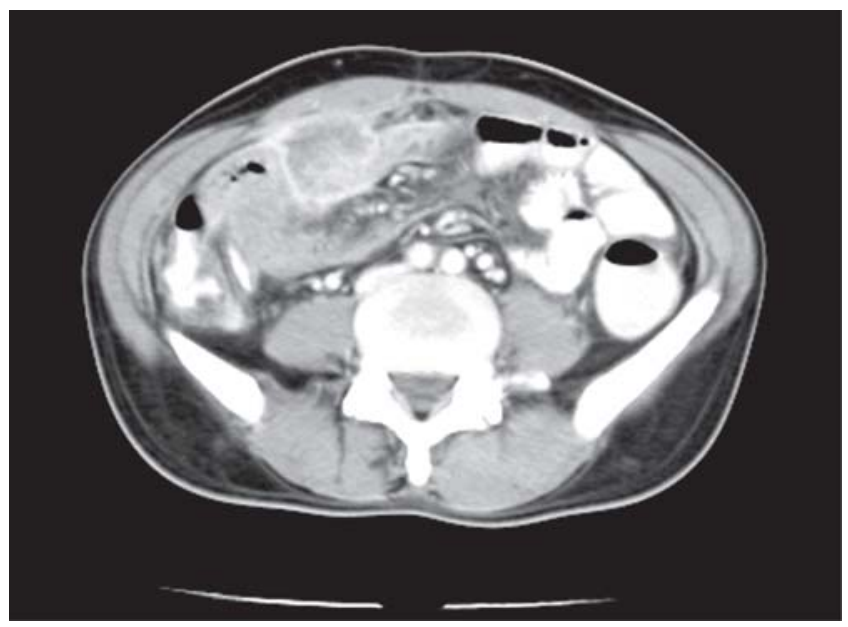

Figure 4. CT scan of brain showing hypodense cystic lesion with mural nodule suggestive of tubercular abscess.

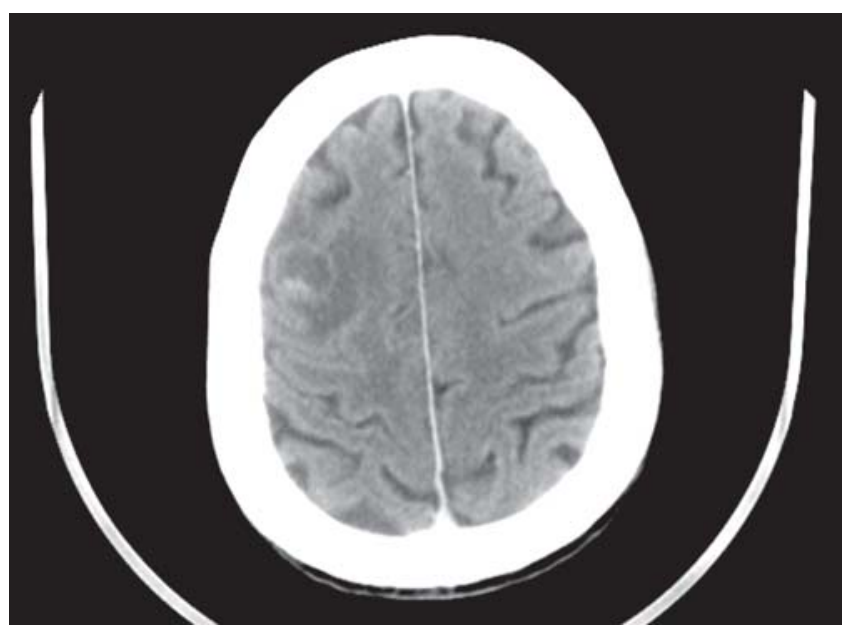

tuberculosis is 5-8\% in presence of dual infection [2]. In HIV infection because of defect in cell mediated immunity organisms multiply rapidly and multiple organs are involved. Significant proportion of tuberculosis infection in AIDS occurs from fresh infection rather than reactivation. Among various forms of tuberculosis in HIV infection, pulmonary involvement is most common followed by lymphnodes. Pleural effusion is found to be relatively common [2]. During the early HIV infection, upper lobe infiltrates and cavitations are common, in late stage miliary infiltrates and diffuse involvements are common. Lymph node involvement may be acute resembling pyogenic adenitis. Other structures involved in late stage are brain, liver, spleen, bonemarrow, soft tissues and testes. Diagnosing tuberculosis may be difficult because of common atypical presentation, many other opportunistic infections that may mimic tuberculosis, atypical radiographic features, 
increased smear negativity, absence of typical granuloma in histopathology and negative Mantoux test.

The pattern of involvement of tuberculosis in HIV infection varies widely. Maniar et al. [7] in a study of 8640 HIV cases from India reported that TB was commonest opportunistic infection in 8078 cases (93.5\%); out of which 3393 (42\%) had pulmonary, 3514 (43.5\%) had extrapulmonary TB and 1171 (14.5\%) had disseminated disease. Ige $\mathrm{OM}$ et al. [8], in a study of pattern of tuberculosis in HIV co infection from Nigeria, reported pulmonary involvement in $78.6 \%$ of the patients, meningial involvement in $7.8 \%$, spine involvement in 6.8\%, lymph node involvement in $4.1 \%$, the other organ (abdomen, pericardial and miliary) involvement in less than $3 \%$ of cases.

In abdominal tuberculosis lymphadenopathy, iliocecal and peritoneal involvement are common. Maniar et al. [7] reported that the commonest form of abdominal involvement is retroperitoneal, mesentric, periportal and peripancreatic lymph node enlargement followed by splenic, liver deposits and iliocecal involvement. Other reported forms of abdominal tuberculosis in HIV are acute peritoneal involvement, hepatic abscess, splenic abscess, pancreatic involvement, abdominal wall and psoas muscle abscess. Ayegnon KG et al. [3] described four cases of acute peritoneal tuberculosis in HIV infection mimicking acute appendicular peritonitis and presenting as acute abdomen. Lupatkin $\mathrm{H}$ et al. [4] observed five cases of large tubercular abscesses located in the liver, abdominal wall, psoas muscle, mediastinum, and peripancreatic areas. In HIV individuals who have fever and localized pain or swelling in the abdomen, tubercular abscess has to be considered and the formation of tuberculous abscesses appears to be a common complication of tuberculosis. Reichel C et al. [9] reported two cases of splenic abscess and suggested that the hypoechoic/hypodense lesions in the spleen of HIV positive patients are highly suggestive of disseminated tuberculosis. Roig RP [10] et al. reported two cases of hepatic tuberculous abscesses in patients with HIV infection, stressing the rare sites of involvement. Nos P et al. [11] described isolated mesentric tuberculosis as a first manifestation of HIV infection where patient presented with fever and painful abdominal swelling. This patient had huge mesenteric abscess with thick capsule and multiple septations which is typically suggestive of tubercular abscess. There was no associated abdominal lymph node enlargement. The mesentric aspirate was caseous material suggestive of abscess. The lesion was extending anteriorly up to rectus muscle and mild thickening of bowel loops of distal jejunum and ileum were also noticed. In presence of any of the abdominal organ involvement in HIV individuals, diagnosis of tuberculosis has to be considered. Careful evaluation of other organs for occult tubercular involvement has to be done. We emphasize considering the diagnosis of tuberculosis even in the absence of pulmonary and lymph node involvement.

Neuro tuberculosis in HIV infection may present as meningitis, tuberculoma and tubercular abscess. 10 to $20 \%$ of cases of AIDS-related extrapulmonary tuberculosis involve the CNS. Focal brain lesions include tuberculomas and cerebral abscesses. The incidence of intracerebral tuberculomas in patients with HIV ranges from 1-10\%; in these patients, cerebral abscesses are much less frequent. Vidal JE et al. [5] reported three cases of tuberculous brain abscess in AIDS patients and reviewed nine similar cases. Excepting one, all patients had single lesion in CT scan. Five patients had extra cerebral involvement predominantly pulmonary and lymph node involvement. None of the patients had associated abdominal involvement. Corti et al.[6] described a case of tuberculous brain abscess and emphasized the early need for stereotactic biopsy or surgical excision. Neuro radiological findings of tubercular abscess are hypodense center, peripheral ring-enhancing, mass effect and marked edema of the white matter. The presence of single lesions with a thick capsule, ring enhancement and lobular contour, make the diagnosis of tuberculous abscess more likely. Tuberculous abscesses are generally single brain lesions, larger than tuberculomas, and evolve more rapidly in comparison. This patient had single enhancing hypodense lesion with perilesional edema and mass effect due to tubercular abscess. Biopsy was not done as the CT scan was typically suggestive of tubercular abscess.

The diagnosis of tuberculosis was established from fine needle aspiration cytology (FNAC) from mesenteric lesion and bonemarrow aspiration smear, which were strongly positive for AFB. The polymerase chain reaction (PCR) for tuberculosis was also positive. Brown et al. [12] emphasized the role of fine needle aspiration cytology in the diagnosis of clinically unsuspected extrapulmonary tuberculosis. In advanced immunosuppression and disseminated tuberculosis, atypical cytological features are common.

In conclusion, we described a case of disseminated tuberculosis with simultaneous mesenteric and brain abscess with involvement of bowel and bonemarrow. Absence of pulmonary and lymphnode involvement is unusual, even in late stage of HIV infection. Aspirates from mesentric abscess and bone marrow were positive for AFB. Literature regarding various ways of presentation abdominal tuberculosis and tuberculous brain abscess in HIV infection is reviewed.

\section{References}

1. Alex S., Jose M.G. Mycobacterial Infection in HIV- infected Patients. In:Jonanlhan C, William G.P., eds. Infectious Disease. Philadelphia: Mosby: Elsevier, 2004: 1303-10.

2. Srikanth T., Jai P.N. Tuberculosis and Human Immunodeficiency Virus Infection. In: Sharma S.K., Mohan A. eds. Tuberculosis. New Delhi: Jaypee, 2004: 404-12. 
3. Ayegnon K.G.,Kouadio K.G.,Kouadio L.N.etal. Acute peritoneal tuberculosis in HIV infected patients, study of 4 cases in Abidjan, Cote-d'Ivoire. Bull Soc Pathol Exot 2006; 99: 177-9.

4. Lupatkin H., Bruan N., Flomenberg P., Simberkoff M.S. Tuberculous abscess in patients with AIDS. Clin Infect Dis 1992; 14: 1040-4.

5. Vidal J.E., Penalva de O.A.C., Bonassern F.F.etal. Tuberculous brain abscess inAIDS patient's report of three cases and literature review.Int J Infect Dis. 2005; 9: 201-7.

6. Corti M.E., Villafane M.F., Yampolsky C.G., Schtirbu R.B. Brain abscess due to mycobacterium tuberculosis in a patient with AIDS: report of a case and review of literature. Int J Infect Dis. 2005; 9: 225-7.

7. Maniar J.K., Kamath R.R., Mandalia S. etal.HIV and tuberculosis: partners in crime Indian J Dermatol Venereol Leprol. 2006; 72:276-82.
8. Ige O.M., Sogaolu O.M., Ogunlade O.A. Pattern of presentation of tuberculosis and the hospital prevalence of tuberculosis and HIV co-infection in University College Hospital, Ibadan: a review of five years (1998 - 2002). Afr J Med Med Sci. 2005; 34:329-33.

9. Reichel C.,Theisen A.,Rockstroh J.K. etal. Splenic abscesses and abdominal tuberculosis in patients with AIDS. Z Gastroenterol. 1996; 34:494-6.

10. Roig R.P., Perez R.L., Navarro I.V. etal. Liver tuberculous abscess in patients with human immunodeficiency virus infection: report of 2 cases and review of the literature Rev Clin Esp. 1995; 195:89-91.

11. Nos P.,Ricart C.,Garcia E. etal Isolated mesenteric tuberculosis as the first manifestation of AIDS. Rev Esp Enferm Dig. 1992;82:59-60.

12. Brown H.M., Abbitt P.L., Wilkinson E.J. Diagnosis of clinically unsuspected extrapulmonary tuberculosis by fine needle aspiration: a case report. Acta Cytol. 2001; 45:1032-6. 